\title{
La tradición del conocimiento simbólico en el pensamiento de Charles S. Peirce
}

\author{
JAVIER LEGRIS \\ Universidad de Buenos Aires \\ Consejo Nacional de Investigaciones Científicas y Técnicas
}

DOI: $10.36446 /$ rlf2020151

I 253

Resumen: La existencia de puntos de contacto entre la teoría de los signos de Peirce y los argumentos de Leibniz en favor del conocimiento simbólico está fuera de duda. El objetivo de este trabajo consiste en ubicar con precisión el pensamiento de Peirce dentro de la tradición del conocimiento simbólico en ciencias formales. El punto de partida es la crítica de Peirce a la noción cartesiana de intuición (alrededor de 1869), en la que se destaca el conocimiento mediante manipulación de signos. A continuación, el trabajo se concentrará en el concepto de iconicidad, tal como surge en la teoría de los signos de Peirce, y el papel que desempeña en la caracterización de las ciencias formales. El concepto de iconicidad se relacionará con las funciones subrogativa y ectética del conocimiento simbólico, pero también se subrayará la esencial función analítica que cumplen los íconos. Sobre esta base, se sostendrá que, si bien el pensamiento de Peirce se nutre de la tradición del conoci- 
miento simbólico, con su concepción icónica de las ciencias formales va finalmente más allá de esta tradición.

Palabras clave: razonamiento diagramático, conocimiento simbólico, Charles S. Peirce, filosofia de las ciencias formales.

\title{
The Tradition of Symbolic Knowledge in Charles S. Peirce's Thought
}

\begin{abstract}
The existence of links between Peirce's theory of signs and Leibniz's arguments in favour of symbolic knowledge is beyond doubt. The aim of this paper is to place Peirce's thought in the tradition of symbolic knowledge in formal sciences. The starting point is Peirce's criticism to the Cartesian notion of intuition. Hereafter, the paper will focus on Peirce's notion of iconicity according to the theory of signs, and its role in the characterization of formal sciences. This notion of iconicity will be related to the subrogative and ecthetic function of symbolic knowledge, but the essential analytic function of icons will be stressed too. On this basis, it will be argued that, even if Peirce's thought is nourished by the tradition of symbolic knowledge, his iconic conception of formal sciences goes beyond this tradition.
\end{abstract}

Key-words: diagrammatic reasoning, symbolic knowledge, Charles S. Peirce, philosophy of formal sciences.

\section{Introducción}

Charles S. Peirce (1839-1914) se cuenta entre los fundadores tanto de la lógica matemática como de la teoría de los signos (semiótica) en su forma moderna. Su pensamiento tuvo uno de sus ejes en la función que cumplen notaciones, figuras y otro tipo de signos en la producción de conocimiento científico (en especial en ciencias formales). El objetivo de este trabajo consiste en situar el pensamiento de Peirce (o al menos la parte más relevante) dentro de la tradición del conocimiento simbólico en la metodología de las ciencias formales, que se hace explícita a partir del pensamiento de Leibniz. ${ }^{1}$

\footnotetext{
${ }^{1}$ Sin duda, hay antecedentes en la teoría de los signos de la Edad Media y en los orígenes del álgebra a comienzos de la Edad Moderna.
} 
Se sostendrá que la idea de conocimiento simbólico, en sus rasgos salientes, se cuenta entre los pilares sobre los que Peirce construyó su pensamiento y es una parte importante del aparato conceptual que Peirce adoptó del pensamiento de Leibniz. ${ }^{2}$ No obstante, la construcción de sistemas diagramáticos para la lógica, acometida por Peirce en el período maduro de su pensamiento, da cuenta de una función analítica de los signos que lo aparta de aquella tradición y lo lleva a buscar nuevas perspectivas.

Dado que el concepto de conocimiento simbólico no puede ser caracterizado con entera precisión y de un modo unívoco en sus orígenes leibnizianos, la tradición que le siguió lo desarrolló en diferentes direcciones, aunque siempre focalizada en los sistemas de signos como vehículos de conocimiento. El concepto de conocimiento simbólico aparece formulado explícitamente por G. W. Leibniz usando diferentes expresiones (cognitio symbolica, cogitatio symbolica, cogitatio caeca) en diferentes contextos y sin darle una formulación teórica stricto sensu. Su recepción posterior depende, en gran medida, del ensayo Meditationes de cognitione, veritate et ideiis, que fue publicado en noviembre de 1684 en las Acta Eruditorum. En este trabajo, Leibniz oponía el conocimiento simbólico al conocimiento intuitivo: el conocimiento es simbólico cuando la complejidad del fenómeno cuyas propiedades se pretenden conocer es tan grande que no es posible tener una intuición de ellas, de modo que la idea de conocimiento simbólico surge en oposición al conocimiento intuitivo. Leibniz proporciona el ejemplo del quiliógono, un polígono de mil lados, que no es factible construir como una figura geométrica, pero al cual puede hacerse referencia mediante un signo. Otros ejemplos están vinculados con el desarrollo del álgebra y del cálculo infinitesimal.

En algunos textos matemáticos de juventud, Leibniz supone que los signos para los infinitésimos no tienen una función designativa, sino que son introducidos para resolver problemas del propio cálculo. Los infinitésimos son entidades ficticias, aunque una "fiction bien fondée" (una ficción bien fundada), pero de hecho se opera de manera consistente con los signos que los representan. (La consideración de los infinitésimos como entidades ficticias está vinculada con su construcción geométrica. Una discusión pormenorizada del problema puede verse en el capítulo 2 de Raffo Quintana 2019 y la relación entre ficciones y conocimiento simbólico es tratada en Raffo Quintana 2020). Estos ejemplos muestran que, expresado desde una perspectiva actual, una de las funciones del conocimiento simbólico surge de

${ }^{2}$ La presencia del pensamiento de Leibniz en la obra de Peirce ha sido estudiada por Max Fisch (véase Fisch 1986), pero en tiempos más recientes se han examinado con detalle los aspectos semióticos (véase inter alia Bellucci 2013 y Fabbrichesi 2011). 
justificar el empleo de determinados signos para realizar cómputos cuyo resultado representa un conocimiento que no sería realizable de facto mediante los sistemas de signos a disposición. Así, el conocimiento simbólico se vuelve necesario debido a las limitaciones cognitivas de los seres humanos y tiene una función pragmática. ${ }^{3}$

La propiedad de symbolicus, aplicada al conocimiento o al pensamiento, se puede entender con el significado de "por medio de signos" o "semiótico" (Leibniz en general emplea el término latino character con el mismo significado que tiene la palabra "signo"). Así, el conocimiento simbólico puede describirse de un modo muy general como el conocimiento obtenido por medio de un sistema de signos, sin tener en cuenta aquello que los signos designan. Esto se manifiesta en la expresión "pensamiento ciego" (cogitatio caeca) que Leibniz usa como sinónimo de conocimiento simbólico, consistente en la manipulación de los signos sin dejar lugar a aspectos intuitivos. Esto permite hablar de una mecanización del razonamiento. De acuerdo con un conjunto de reglas del sistema se producen signos complejos a partir de un conjunto de signos básicos. Esta es una innovación metodológica: a la manipulación de signos se le asigna una posición destacada en el conocimiento humano. Los sistemas de signos proporcionan procedimientos tanto de demostración como de decisión y las demostraciones se entienden como calculus dentro de un sistema de signos. Estas ideas están presentes, por ejemplo, en el prefacio a la ciencia general (Science générale) de 1677 (Leibniz C 155).

Una descripción de los rasgos centrales del conocimiento simbólico a partir de un análisis de los textos de Leibniz puede encontrarse en Esquisabel (2012: 18 ss.), donde se determinan sus rasgos y sus diferentes funciones. Allí se menciona también un concepto "amplio" de conocimiento simbólico que resulta de considerar que las estructuras de signos exhiben algo ad oculos (Esquisabel 2012: 26 s.), tal como sucede con diagramas y figuras. Se trata de signos que hacen visibles o exhiben una estructura, a los que Leibniz llamaba ectéticos (siguiendo a Joachim Jungius). Leibniz deja en claro la naturaleza composicional y, por tanto, analítica, que tiene este tipo de signos y en ocasiones incluye entre los signos ectéticos todo tipo de notaciones y sistemas de signos que no se corresponden con el lenguaje ordinario (véase Esquisabel 2012: 27).

En consecuencia, pueden distinguirse en sus raíces leibnizianas dos variedades del conocimiento simbólico: (1) un conocimiento obtenido por

\footnotetext{
${ }^{3}$ Sin duda, uno de los interrogantes que surge aquí para el lector actual es el problema de la consistencia del sistema de signos ampliado. Históricamente, este problema está implícito en la disputa por la corrección del cálculo infinitesimal a finales del siglo XVII y el siglo XVIII.
} 
la operación mecánica de signos de acuerdo con reglas (sentido estricto); (2) conocimiento obtenido por la visualización de la estructura de uno o más signos. En un afán simplificador, puede hablarse de una variedad computacional y otra estructural. Como se verá más adelante, la distinción no será todo lo nítida que se quisiera, pues hay sistemas de signos que pueden ubicarse dentro de ambas variedades. También se verá que la función analítica será propia de la segunda variedad, aunque también puede encontrarse en la primera. Esta distinción será relevante al examinar el caso de Peirce con su teoría de los signos.

Las ideas de Leibniz inician una amplia tradición en la metodología de las ciencias formales, que puede ser estudiada siguiendo la doble perspectiva de la historia de las ideas y de la historia de las influencias (para esta distinción, véase Angelelli 1983). Esta tradición se desarrolla siguiendo diferentes interpretaciones de las ideas originales y comienza a manifestarse en el pensamiento inmediatamente posterior a Leibniz. Dan cuenta de ello las obras de Christian Wolff, Alexander Gottlieb Baumgarten, Georg Friedrich Meier, Joachim Georg Darjes, Johann Heinrich Lambert, Salomon Maimon o incluso el Kant precrítico.

\section{Conocimiento simbólico y los orígenes del álgebra de la lógica}

$\beth_{\text {genes de la lógica matemática. Durante el siglo XIX, las ideas de }}^{\text {n forma subteránea, esta tradición también pervive en los orí }}$ Leibniz influyeron en algunos matemáticos alemanes que participaron en la génesis de la lógica matemática (sobre todo en cuestiones que tienen que ver con el álgebra, véase Peckhaus 1997: 233 ss.) y son bien conocidas las referencias a Leibniz que Frege hace al presentar su programa de una escritura conceptual (véase al respecto Legris 2012).

La forma en que las ideas de Leibniz sobre el método en las ciencias formales se transmiten en el Reino Unido es más indirecta (e implica un detour por la matemática en la Francia del siglo XVIII que dio importancia al papel de los signos en la formulación de teorías matemáticas, en particular el análisis, vale mencionar simplemente el caso de Condillac). Gracias al esfuerzo de la Analytical Society (creada en 1812), la Universidad de Cambridge adoptó la notación leibniziana para el cálculo infinitesimal, en lugar del cálculo de fluxiones de Newton. Un suceso más importante para este trabajo es la formulación del álgebra simbólica que George Peacock (miembro de la Analytical Society) hace en su Treatise on Algebra de 1830. Pocos años después formula Peacock el "principio de permanencia de las 
formas equivalentes (equivalent forms)", según el cual una equivalencia algebraica lo es con independencia de la interpretación de sus signos (Peacock 1834: 198 s.). Un poco después, el matemático escocés Duncan Gregory definió el álgebra simbólica como "la ciencia que se ocupa de la combinación de las operaciones definidas no por su naturaleza [...] sino por las leyes de combinación a las que están sujetas" (Gregory 1839: 208).

En su libro The Mathematical Analysis of Logic, publicado en 1847, Boole, discípulo de Gregory, aplicó las ideas del álgebra simbólica al campo de la lógica deductiva. Boole sostenía explícitamente que "la validez de los procesos de análisis" dependía exclusivamente de "las leyes de combinación" (Boole 1847: 3). Esta idea se continúa y se profundiza en su obra The Laws of Thought, de 1854, donde se ocupa de describir el método al que llama "razonamiento simbólico", que permite estudiar propiedades de sistemas algebraicos de manera independiente de contenidos, y dando la libertad de aplicarlo a dominios diversos (véase Boole 1854: 67).

No es difícil ubicar esta metodología propuesta por Boole dentro de la tradición del conocimiento simbólico. Si bien la mención a "entidades ficticias" es reemplazada por la apertura a posibles casos de aplicación, los resultados se obtienen de manera "ciega". El "simbolismo operativo" es considerado un "instrumento del razonamiento" (véase Legris 2012).

Ernst Schröder llevó a cabo un prolijo y cuidadoso trabajo de sistematización del álgebra de la lógica en la última década del siglo XIX. En la introducción al volumen primero de sus Vorlesungen über die Algebra der Logik presenta sus ideas acerca de la lógica, los sistemas de signos y el cálculo formal. Influido por Peirce, esboza una fundamentación semiótica de la lógica. Para Schröder el objeto de la lógica era el pensamiento (Denken), siendo su objetivo final la adquisición de conocimiento (véase Schröder 1890: 1). Este pensamiento "reducido a su expresión más estricta" se da bajo la forma de un cálculo (Schröder 1890: 10) y toda deducción se lleva a cabo por medio de un cómputo (rechnerisch). Como consecuencia, la deducción "no puede rehuir a la poderosa ayuda de la percepción", que es la observación de los signos (Schröder 1890: 10). Los ecos del conocimiento simbólico, en su forma más estricta, resuenan vigorosamente en sus palabras. La idea de "hacer perceptible" el pensamiento mediante su manipulación es la función del cálculo.

En su juventud, Peirce comenzó a investigar el álgebra de la lógica, continuando la línea de trabajo iniciada por Boole, aunque con el énfasis puesto en desarrollar una lógica de relaciones basada en las aportaciones de De Morgan. Su primera contribución sobresaliente, que tendrá un gran impacto, está en la notación que introduce. Peirce objeta la idea algebraica de presentar la estructura lógica de enunciados a través de ecuaciones y, así, propone reemplazar el signo de identidad por la "pata de gallo" (el "crow foot" 
' $-<$ ), que refleja una relación de orden parcial. Esta relación puede tener diferentes interpretaciones. La primera corresponde a la ilación, término que él toma de la lógica del siglo XIV y que se refiere a la relación entre las premisas y la conclusión en un razonamiento deductivo. En su trabajo de 1870 "Description of a notation for the logic of relatives, resulting from an amplification of the conceptions of Boole's calculus of logic" (CP 3.45-148), Peirce introduce el signo de ilación con esta interpretación (CP 3.47). Sin embargo, en otros trabajos - por ejemplo, "On the algebra of logic" (CP 3.154-251) de 1880- el signo recibe interpretaciones alternativas: (i) la cópula de los silogismos, (ii) inclusión entre conjuntos, (iii) la relación de implicación entre enunciados o (iv) el condicional como función de verdad (véase la discusión en Dipert 1981: 579 ss.). Aquí habría un esbozo de la idea de proporcionar diferentes interpretaciones a un sistema de signos. ${ }^{4}$ En todo caso, Peirce introduce el crow-foot como consecuencia de su "filosofia de la notación", según la cual, entre otras cosas, debe haber una relación intrínseca entre los signos y las características de sus designados. Esto cobra una especial importancia en el caso de la notación lógica y matemática. La naturaleza de esta relación está determinada por la teoría de los signos desarrollada por Peirce.

\section{Conocimiento simbólico en la teoría de los signos de Peirce}

T a idea de conocimiento simbólico aparece de un modo más ex-

L plícito al excavar en las profundidades de la teoría de los signos desarrollada por Peirce (es decir, su semiótica o "semeiótica", como Peirce prefería denominarla). En definitiva, es en virtud de su teoría de los signos que Peirce puede ubicarse dentro de la tradición del conocimiento simbólico iniciada por Leibniz. En ocasiones, Peirce se refiere a su semiótica general como "Lógica" en un sentido amplio de la palabra (véase CP 1.444, 2.93). Originalmente, Peirce concibió su teoría de los signos como una teoría acerca de la mente y el conocimiento, cuyos rasgos se manifiestan en el uso de los signos.

Peirce tomó el término "semiótica" del Ensayo de Locke, donde designa "otro tipo de lógica" en el que las ideas son consideradas como signos

\footnotetext{
${ }^{4}$ Esta idea ya está presente en Boole pero fue desarrollada en forma explícita y general tiempo después por Schröder en su Lehrbuch der Arithmetik und Algebra de 1873 al determinar la subsunción como el caso lógico de la relación más abstracta y formal de subordinación del "álgebra absoluta", que incluía un "algebra formal" junto con sus interpretaciones (véase Legris 2015).
} 
(Locke Essay, B. IV, cap. XIII). Más allá de este hecho, una influencia directa de la obra de Leibniz resulta enteramente plausible. En su juventud, Peirce comenzó a recolectar ideas para un "Vocabulario Filosófico" (que cristalizaría mucho más tarde en las entradas que escribió para el Dictionary of Philosophy and Psychology [1901-1902], editado por Baldwin). En los esbozos de este Vocabulario aparecen típicas nociones leibnizianas para cualificar formas de conocimiento tales como claro y oscuro, adecuado e inadecuado, y, finalmente, intuitivo y simbólico (llamando a este último también "ciego" o "supositivo"). La fuente que adoptó Peirce es la edición publicada por Johann Eduard Erdmann de la obra de Leibniz en 1840 (véase Erdmann). Muchas de las referencias corresponden al trabajo antes mencionado Meditationes de cognitione, veritate et ideis. Peirce presentó la clasificación de los signos entre íconos, índices y símbolos en su decisivo artículo "On a New List of Categories" de 1867 (CP 1.558 s.). En un borrador para este trabajo fechado el año anterior (MS 720), ${ }^{5}$ él citaba el "célebre pasaje" del quiliógono como un ejemplo del uso "ciego o simbólico" de los signos y justificaba su propia elección del término "símbolo" para este tercer tipo de signos (véase Fisch 1986: 253) ${ }^{6}$

Todo esto lleva a considerar la semiótica de Peirce como una continuación del pensamiento moderno. Esto se hace más evidente al descubrir que el recurso a ideas y argumentaciones leibnizianas desempeñó un papel importante en el rotundo anticartesianismo que Peirce sostuvo desde un comienzo y que fue determinante para su filosofia. Entre 1868 y 1869 Peirce publicó en el Journal of Speculative Philosophy tres trabajos, conocidos comúnmente como la "cognition series". En ellos Peirce introdujo muchos de los problemas centrales que serían una constante a lo largo de su reflexión filosófica. En particular, dio razones para afirmar su antipsicologismo y su concepción del conocimiento como un proceso esencialmente inferencial. Peirce sostenía allí que la duda universal cartesiana o la conciencia individual no pueden servir como puntos de partida de una concepción filosófica.

En el primero de los trabajos de esta serie, Peirce rechazaba la actitud cartesiana de basar el conocimiento en intuiciones y comenzaba caracterizando las intuiciones como cogniciones (cognitions) que no están determi-

\footnotetext{
${ }^{5}$ Los manuscritos se indican según la numeración en la colección que se encuentra en la Universidad de Harvard: Charles S. Peirce Papers, 1787-1951 (MS Am 1632), Houghton Library, Harvard University (https://id.lib.harvard.edu/ead/hou02614/).

${ }^{6}$ De hecho, Peirce tenía una enorme estima por el pensamiento de Leibniz y lo consideró un antecedente inmediato de su pragmatismo. En un manuscrito, Peirce señalaba que con su concepción de conocimiento simbólico Leibniz habría llegado al "umbral de la teoría pragmática" (véase Bellucci 2013: 31 y s.).
}

JAVIER LEGRIS - La tradición del conocimiento simbólico en el pensamiento de... I 253-271 
nadas por otras cogniciones, esto es, que están "determinadas por algo fuera de la conciencia” (CP 5.213). En el mismo pasaje, recurría a una analogía con los juicios (judgements); las intuiciones se comportaban como "premisas que no son ellas mismas conclusión", de modo que, al igual que estas, requería de una fundamentación externa: el "objeto trascendental".

En la primera sección del segundo artículo de la serie,"Some Consequences of Four Incapacities", continúa su refutación del "espíritu del cartesianismo", al que considera una base equivocada para la ciencia moderna, y señala específicamente "cuatro errores" que presenta en relación con el conocimiento. Entre las respuestas o enmiendas a estos errores, Peirce afirma uno de los principios básicos de su pensamiento: "No tenemos la capacidad (power) de pensar sin signos" (CP 5.265). El nexo con la tradición del conocimiento simbólico resulta obvio.

Peirce sostenía que el conocimiento se basaba en inferencias (y no en intuiciones) de modo que los juicios o enunciados tienen un papel central en los procesos gnoseológicos y propone "reducir toda acción mental a las fórmulas de la inferencia válida" (CP 5.279). En este punto es donde introduce la conocida división de los razonamientos en deducción, inducción e hipótesis (lo que llamará más tarde abducción).

Por lo demás, cabe notar que Peirce rechazaba el psicologismo de John Stuart Mill, dado que la lógica estudia los "productos de pensamiento", tales como los enunciados expresados en el lenguaje. En la primera de las conferencias pronunciadas en Harvard en 1865, Peirce afirmó:

La perspectiva psicológica es que estas formas son realizadas únicamente en el pensamiento [...] La perspectiva no psicológica (unpsychological) es que estas son formas de todos los símbolos, tanto internos como externos, pero que existen únicamente en virtud de un pensamiento posible. (Peirce $W$, vol. 1,165$)$

A continuación, añadió:

Yo digo que la forma lógica ya está realizada en el símbolo mismo; los psicologistas dicen que esta solo se realiza cuando el símbolo es comprendido. (Peirce $W$, vol. 1, 165-166)

Por lo tanto, para Peirce la forma lógica de un razonamiento se constituye como un objeto semiótico. Desde ya, esta es una consecuencia decisiva para su filosofia de la lógica.

La concepción del conocimiento que Peirce defiende en este período puede resumirse del siguiente modo: si todos los pensamientos son 
signos, entonces el marco en el cual se analizan los signos puede aplicarse al análisis de fenómenos mentales. De acuerdo con esto, los estados mentales son análogos a enunciados y una acción mental es análoga a hacer inferencias. Las ideas de la teoría moderna del conocimiento son reemplazadas por juicios (al menos en el sentido de que son los elementos básicos) y lo que se veía como mecanismos de asociación de ideas son ahora reglas de inferencia.

Ahora bien, la tesis de que el pensamiento únicamente se lleva a cabo por medio de signos puede interpretarse en un sentido más fuerte: todos los pensamientos deberían ser entendidos como signos. En su artículo sobre las "four incapacities", Peirce habla directamente de "signos-pensamiento" ('thoughtsigns'):

[...] siempre que pensamos tenemos ante la conciencia alguna sensación, imagen, concepción u otra representación que sirve como signo [...] Ahora bien, un signo tiene como tal tres referencias (references): $1^{\circ}$, es un signo para algún pensamiento que lo interpreta; $2^{\circ}$ es un signo de algún objeto de que es equivalente en ese pensamiento; $3^{\circ}$, es un signo en algún aspecto o cualidad, que lo pone en conexión con su objeto. (CP 5.283, OFE 82 s.)

En este pasaje aparece bosquejado el proceso de semiosis (en parte sobre la base de conceptos propios de la filosofía moderna que a Peirce le será difícil abandonar). Se habla del signo-objeto (sign-object), signo-vehículo (sign-vehicule) o representamen, de su referencia o designatum, del interpretante y también de lo que Peirce llamó "fundamento" (ground) del signo (el modo de designar que tiene el representamen). Este proceso se aplica a signos de cualquier tipo: natural, convencional o lingüístico, y surge de la relación entre los tres elementos que constituye la base de su teoría de los signos. Así, "conocimiento semiótico" es una expresión que se aplicaría al menos a una buena parte de la teoría peirceana de los signos: la semiótica asume muchas de las funciones asignadas a la teoría del conocimiento y mucho de lo discutido sobre el conocimiento simbólico (si no todo) queda subsumido dentro de la semiótica.

Otra faceta de la oposición de Peirce al pensamiento cartesiano se encuentra en su atención a los aspectos sociales del conocimiento. En ocasiones, Peirce deja en claro la prioridad de lo social respecto del sujeto o el yo (Self, véase CP 1.673). El conocimiento científico es una tarea social y no meramente individual que es llevada a cabo por una "comunidad de investigadores" (community of inquirers). La idea de una comunidad de investigadores aparece esbozada en el trabajo "The Fixation of Belief”, de 1877, en relación con el "método de la indagación" (véase Liszka 1978). Al año siguiente, 
Peirce menciona un "sentimiento social" como un supuesto de la lógica (entendida en un sentido amplio) y que tiene que ver con la continuidad en el tiempo (y también en el espacio) de la investigación (CP 2.655, OFE 1,197). El conocimiento humano stricto sensu es algo que se obtiene plenamente a través de la comunicación entre todos los miembros de una comunidad que no tiene límites precisos, ni espaciales ni temporales. Resulta obvio que esta comunicación es posible únicamente por medio de sistemas de signos.

\section{La teoría madura de los signos: diagramas y conocimiento}

Deirce advirtió que una concepción diádica del signo no es completa: que una entidad sea un signo implica algo más que estar en una relación diádica de designación respecto de alguna otra entidad. Este "algo más" es el hecho de ser interpretado como signo de esa entidad. Lo que se entiende por significado de un signo descansa en el hecho de que los usuarios del signo pueden interpretarlo de un modo específico (recuérdese la importancia de la noción de comunidad en su filosofia). Así, para Peirce la relación de "ser un signo" es triádica: $X$ es un signo de $Y$ para $Z$. Como se lee en el célebre pasaje de su Gramática especulativa de 1893:

Un signo, o representamen, es algo que está para alguien en lugar de algo en algún respecto o capacidad. (CP 2.228)

La relación entre un signo y lo designado depende de la comprensión de que una entidad es un signo de otra entidad. Además de su función representativa, el signo-objeto tiene funciones importantes en su relación material con el designado (entre las cuales está el ground, "fundamento", del signo-vehículo; véase CP 5.287).

Ahora bien, Peirce concibió su distinción categorial entre "primeridad", "secundidad" y "terceridad" ("Firstness", "Secondness", "Thirdness") que atraviesa todo su pensamiento y lo determina. No es este el lugar para ocuparse en detalle de esta distinción básica, pero basta con destacar que implica un orden de complejidad, y que se manifiesta también en el plano semiótico: la teoría de los signos de Peirce clasifica los signos en términos de tricotomías. Una de estas clasificaciones tiene que ver con la relación entre el signo-vehículo y el designatum. Se trata de la clasificación en ícono, índice y símbolo (icon, index, and symbol), a la que considera "muy importante" pues "son indispensables en todo razonamiento" (CP 1.369). Típicamente, se la puede caracterizar del modo siguiente (véase el cap. 3 de su Gramática especulativa, CP 2.247 ss.). Los íconos representan su objeto de acuerdo con su 
forma o estructura (en este sentido se habla de una semejanza entre el signo y su designado). Los índices se conectan de manera directa con lo que designan: un índice "refiere al objeto que denota en virtud de que es realmente afectado por aquel objeto" (CP 2.248). Piénsese en la huella de un pie en la playa o en los nombres propios del lenguaje ordinario. Finalmente, un símbolo designa "en virtud de una ley, usualmente una asociación de ideas generales". Esta ley determina la interpretación del signo, que hace que el símbolo tenga un designado determinado (CP 2.249). En realidad, estos tres tipos son tres aspectos que se manifiestan en todos los signos: en cualquier signo pueden encontrarse aspectos icónicos, indexicales o simbólicos, pero siempre un aspecto va a preponderar en la interpretación del signo.

La conexión de esta distinción con la tradición de conocimiento simbólico no está exenta de problemas. En efecto, esta distinción abre diferentes modos en los que es posible obtener conocimiento a partir de los signos. En este sentido, conviene hablar mejor de conocimiento semiótico, y se puede conjeturar que tiene un sentido más amplio que la idea prevaleciente de conocimiento simbólico basada en la manipulación de signos.

En este punto, la importancia de esta distinción se hace patente porque hace entrar en escena un concepto que ha tenido un lugar particular en la tradición del conocimiento simbólico. Se trata del concepto de diagrama.Varios autores posteriores a la tradición de Leibniz pusieron de relieve el enorme valor cognitivo que tienen los diagramas para entender de manera visual relaciones y estructuras, especialmente en el caso de la matemática. Este carácter destacado de los diagramas adopta un carácter prominente en Peirce. El marco de la teoría de los signos lleva a una caracterización más precisa de lo que es un diagrama, al incluirse en la categoría de los íconos: "Un diagrama es un ícono de un conjunto de objetos racionalmente relacionados." (MS 293: 11)

En algunos textos en los que Peirce clasifica los íconos, los diagramas aparecen como un tipo dentro de estos; en otros textos los considera equivalentes (véase, por ejemplo, CP 2.277). Sea como fuere, lo importante es que, en cualquier ícono, su estructura ("forma") es esencial para su función semiótica. Los íconos son signos que proporcionan conocimiento a partir del mero examen de su estructura ${ }^{7}$ y permiten acceder a información que va más allá de la indicada por las reglas de su construcción (véase CP 2.279). Siempre que se trata de transmitir una idea, es necesario recurrir a íconos (CP 2.278), y todo enunciado debe contener íconos que se expresan lin-

\footnotetext{
${ }^{7}$ Es por esta razón que se trata de una "primeridad".
} 
güísticamente a través de predicados, de modo que los diagramas no designan objetos sino estructuras.

Estas características tienen importantes consecuencias epistemológicas. Peirce sostuvo que la lógica y la matemática son ciencias esencialmente diagramáticas; esto es lo que permite considerarlas ciencias formales (en el sentido habitual del término). Más concretamente, la deducción es una relación basada en la naturaleza icónica de la forma lógica de enunciados: deducir es manipular diagramas. En su trabajo seminal sobre álgebra de la lógica de 1885, Peirce afirmaba:

[...] todo razonamiento deductivo [...] implica un elemento de observación; a saber, la deducción consiste en la construcción de un ícono o diagrama [...] en experimentar con esta imagen en la imaginación y en observar el resultado para descubrir relaciones desapercibidas y ocultas entre las partes. (CP 3.363, trad. OFR I, p. 273)

Una concepción diagramática de la deducción queda claramente delineada. La deducción incluye acciones sobre diagramas: construir, transformar y experimentar, y la función experimental consiste en la introducción de supuestos expresados icónicamente. ${ }^{8}$ Según el capítulo segundo de su Gramática especulativa (CP 2.227), durante la construcción del diagrama en la imaginación se hacen las modificaciones requeridas por "el estado de cosas hipotético" y se observa si el resultado concuerda con lo que se quiere deducir. ${ }^{9}$

Ejemplos típicos de deducciones diagramáticas son las demostraciones de la geometría euclideana, que incluyen manipulación y transformación de figuras geométricas, y otro ejemplo más conspicuo lo constituyen los diagramas deVenn aplicados a la teoría tradicional del silogismo, donde es posible determinar mediante acciones en un diagrama (que representa la conexión de los tres términos presentes en un silogismo) si el silogismo es válido o no.

Peirce desarrolló a partir de 1896 y hasta su muerte en 1914 un método exclusivamente diagramático, los gráficos existenciales, basado esencialmente en el trazado y manipulación de líneas y elipses que se interpretan como signos para conceptos lógicos que se correspondían con los conceptos

\footnotetext{
${ }^{8}$ Este hecho es un aspecto esencial para el concepto de demostración matemática que formula Peirce, que incluye demostraciones hipotéticas (con supuestos) a las que llama "teoremáticas", véase Peirce NEM 4, 38 y ss.

${ }^{9}$ Como señala enfáticamente en un texto recogido en el vol. I de los New Elements: “¡El mejor pensamiento, especialmente sobre temas matemáticos, se hace experimentando en la imaginación sobre un diagrama u otro esquema!" (NEM 1, 122).
} 
de la lógica de primer orden con identidad (un texto clásico sobre el tema es Roberts 1973). El interés no era didáctico o puramente instrumental, sino enteramente conceptual; los gráficos existenciales eran la forma más adecuada de representar las deducciones y por ello Peirce los consideró su chef d'oeuvre en lógica y como la "lógica del futuro" (véase Roberts 1973:11). Las deducciones se desarrollaban a partir de diagramas que representaban las premisas de un razonamiento y estaban formadas por pasos que resultaban de acciones sobre los diagramas (inscribir, borrar, duplicar, etc.). Sin duda, estos gráficos eran una realización concreta de su concepción diagramática de la lógica.

\section{Iconicidad y análisis}

T a noción de ícono es fundamental, pues determina la concepción Lque Peirce tiene del conocimiento en las ciencias formales. Desde el momento en que dan información en virtud de su estructura (no es necesario ir más allá del signo para obtener información de su designado), los íconos abarcan cualquier notación de naturaleza composicional. En general, los íconos son los signos que resultan informativos al ser analizados. ${ }^{10}$ En unas notas sobre la filosofia científica Peirce los llama "imágenes analíticas" (CP 1.275). Si un signo es cabalmente analizable, sin duda es un ícono. Por ejemplo, la notación algebraica constituye un sistema de íconos y, de hecho, para Peirce las ecuaciones algebraicas son íconos: "una fórmula algebraica es un ícono", afirma Peirce (CP 2.279, y véase también CP 4.424). (Esto ya estaba puesto de relieve en su trabajo de 1885, donde los principios del sistema algebraico reciben el nombre de "íconos".)

De este modo, ya su trabajo en álgebra de la lógica manifiesta una concepción icónica de la lógica y la matemática, y los gráficos existenciales no son otra cosa que una continuación de la notación algebraica haciendo uso de otros recursos de notación. Su finalidad consistía en "separar el razonamiento en sus partes más pequeñas, de modo que cada una de ellas pueda ser examinada en sí misma" ( $M S$ 455, p. 2 - 1903). Es por esto que no debían entenderse como un "cálculo" lógico sino como una manera de "hacer una disección" de las operaciones de deducción en todos sus pasos elementales (véase CP 4.424).

Ahora bien, prima facie, "análisis" significa la descomposición de algo en sus elementos más básicos y se aplica a conceptos en general. Peirce da

${ }^{10}$ Esto es lo que Frederik Stjernfelt ha denominado "iconicidad operacional", véase Stjernfelt 2006. 
como ejemplo la relación de ser abuelo (genérico). "A es abuelo (genérico) de B sí y solo si A es padre (genérico) de alguien que es padre (genérico) de B" (CP 1.294). Esta es la idea más usual de análisis. No obstante, Peirce añade un sentido particular cuando afirma que los gráficos existenciales son "de lejos más perfectos" que el álgebra (CP 4.429), puesto que analizan mejor la forma lógica de los enunciados que aparecen en deducciones. Recientemente se ha encontrado en manuscritos no publicados la justificación que Peirce da a esta afirmación. Hay dos ideales que los gráficos existenciales aspiraban cumplir: (a) tener el menor número posible de signos lógicos y (b) construir de un modo único sus expresiones (véase Bellucci \& Pietarinen 2016: 210 s.). Peirce señalaba expresamente que los gráficos existenciales proporcionaban "el único método" que recurría a un "único signo" para expresar las operaciones lógicas y que "reconocía un único modo de combinación de ideas" (MS 482, "On Logical Graphs" ca. 1897, y MS 490, de 1906). ${ }^{11}$

Independientemente de la realización concreta en los gráficos existenciales, estos ideales conducen a una idea más estricta de análisis como "unicidad de descomposición". En un sistema de signos "perfecto" habrá un modo único de descomponer un signo compuesto en otros más simples y esta descomposición, desde luego, también tendrá un efecto semántico: si la descomposición es única, habrá un modo privilegiado y genuino de acceder a la naturaleza del designado. Ahti-Veikko Pietarinen ha discutido ulteriormente esta noción de análisis (véase Pietarinen 2018).

Finalmente, cabe mencionar un aspecto adicional de los íconos, vinculado con la tradicional idea de semejanza o analogía. La simplicidad de la acción del trazo y la simplicidad topológica del diagrama inscripto tienen semejanza con el carácter elemental del concepto. El scroll de los primeros sistemas de los gráficos existenciales para la lógica de enunciados es un ejemplo típico (el scroll se traza como "una línea curva sin flexión en contrario y en retorno"; véase Legris 2018). En suma, la reducción de signos a un mínimo, la unicidad de descomposición y la simplicidad del signo son aspectos que entran en consideración en la concepción de análisis que Peirce maneja. ${ }^{12}$

Una breve comparación con las ideas de Lambert acerca de la función cognitiva de los diagramas será de utilidad para aclarar los puntos precedentes. Como se mencionó en la primera sección, Johann Heinrich Lambert (1728-1777) continúa las ideas de Leibniz en el siglo XVIII y fue uno de los

\footnotetext{
${ }^{11}$ Este único signo es el scroll de las primeras formulaciones de los gráficos existenciales, que representaba la implicación.

${ }^{12}$ En los gráficos existenciales esta peculiar idea de análisis aparece en relación con la idea de un sistema que exprese la logicidad en un único signo (el scroll, véase Legris 2017).
} 
miembros de la denominada "tradición del conocimiento simbólico" que recibieron su influencia directa. Lambert presentó su concepción del conocimiento científico en la tercera parte de su Neues Organon, de 1764, que lleva por título "Semiotik". Allí se refiere al sistema simbólico perfecto o "científico", que tiene una función naturalmente "subrogativa": trabajar con los signos en lugar de las entidades designadas por ellos. Según Lambert, el álgebra es el modelo más perfecto de sistema simbólico científico, puesto que, entre otras cosas, es un lenguaje de ecuaciones, que contiene un cálculo o cómputo (Lambert 1764, $\$ 35,23$ ). Pero, además, el cálculo puede realizarse sin necesidad de recurrir al significado de los signos mediante la aplicación de reglas para su transformación (Lambert 1764, \54,34).

En el volumen II de esta obra, Lambert añade a esas condiciones de los signos la de tener un máximo de semejanza estructural con los objetos que designa, de modo que su función "subrogativa" se cumple de un modo perfecto (véase Esquisabel 2006: 73). En este punto, Lambert adopta el concepto "amplio" de conocimiento simbólico y lo desarrolla. En efecto, según la función subrogativa, la relación entre signo y designado no requerirá de reglas convencionales; la semejanza entre ambos sería captada de manera directa (véase Lambert $1764, \mathbb{5} 54,33-34, \$ 58,37, \$ 61,39$ ). En estos casos, Lambert habla del signo como un dibujo (Zeichnung) que sirve como metáfora del designado (Esquisabel 2006: 73). En esto parece preanunciar parte de las ideas de Peirce. No obstante, Lambert incluye también la idea de un análisis del significado, puesto que los signos de un sistema simbólico perfecto tienen siempre designación (véase Esquisabel 2006: 72), pero Peirce va más allá de este análisis de significado con su noción de ícono; el significado (formal, estructural) está dado por el ícono mismo.

Esta noción de análisis es el producto de la original idea de iconicidad surgida de su teoría de los signos y de sus supuestos filosóficos. Con ella, Peirce avanza unos cuantos pasos más allá del concepto de conocimiento simbólico. El "conocimiento semiótico" de Peirce tiene esta función analítica, ausente en el "conocimiento simbólico". ${ }^{13}$

En suma, la concepción de Peirce se diferencia de la tradición del conocimiento simbólico en la función que asigna a los signos de la lógica y la matemática. Para Peirce el carácter analítico de los signos icónicos junto con las operaciones efectuadas sobre estos son esenciales para las ciencias formales, de

\footnotetext{
${ }^{13}$ Es difícil evitar hacer una comparación entre la situación de Peirce y la de Gottlob Frege, quien también introduce, en el marco enteramente distinto de su notación conceptual (Begriffsschrift), una idea de análisis semántico que lo lleva a alejarse de la tradición del conocimiento simbólico (véase Legris 2012: 109 y ss.).
}

JAVIER LEGRIS - La tradición del conocimiento simbólico en el pensamiento de... I 253-271 
modo que su perspectiva trasciende cuestiones puramente metodológicas. A la vez, se diferencia también de cualquier variante de formalismo en filosofía de la matemática. Los sistemas de signos icónicos, que pueden tener un alto grado de complejidad e incluir construcciones hipotéticas, son la base de las ciencias formales y lo que conduce más específicamente al conocimiento matemático (tanto en la generación de ese conocimiento como también en su justificación). Es evidente que la filosofía de la matemática que Peirce propuso se construye sobre la base de su teoría de los signos y forma parte de la tradición del conocimiento simbólico, pero sus convicciones filosóficas lo llevaron a avanzar hacia otros territorios. ${ }^{14}$

\section{BIBLIOGRAFÍA}

Angelelli, I. (1983), "Review of Gottlob Frege de Hans Sluga", The Journal of Philosophy, 80 (4): 232-239. DOI: $10.2307 / 2026006$

Bellucci, F. (2013), "Peirce, Leibniz, and the Threshold of Pragmatism”, Semiotica, 195: 331-355. DOI: 10.1515/sem-2013-0030

Bellucci, F. y Pietarinen, A.-V. (2016), "Existential Graphs as an Instrument for Logical Analysis. Part 1: Alpha”, Review of Symbolic Logic, 9 (2): 209-237. DOI: 10.1017/S1755020315000362

Boole, G. (1847), The Mathematical Analysis of Logic: Being an Essay Towards a Calculus of Deductive Reasoning (Cambridge: Macmillan, Barclay and Macmillan).

Boole, G. (1854), An Investigation of the Laws of Thought, on which are Founded the Mathematical Theories of Logic and Probabilities (Londres: Walton and Maberly).

Chapman, P., Stapleton, G., Moktefi, A., Pérez-Kriz, S. y Bellucci, F. (2018) (comps.), Diagrammatic Representation and Inference, Lecture Notes in Computer Science 10871 (Cham: Springer). DOI: 10.1007/978-3-319-91376-6

Dipert, R. (1981), “Peirce's Propositional Logic”, The Review of Metaphysics, 34: 569-595. Esquisabel, O. M. (2006), "Lambert: representación, conocimiento simbólico y dia-

14 Este trabajo fue realizado en el marco de los proyectos PIP 11220170100463CO, ANPCyT PICT 20170506 y UBACYT 20020170100684BA. El trabajo es deudor de las reiteradas y extensas discusiones sobre el concepto de conocimiento simbólico sostenidas a lo largo de la última década con Oscar M. Esquisabel y Abel Lassalle Casanave: también se ha beneficiado con las discusiones sostenidas con Valeria Giardino y Gerhard Heinzmann durante una estadía en los Archives Herni Poincaré (Nancy) en 2018. Las estimulantes observaciones críticas de los árbitros anónimos de la RLF contribuyeron a mejorar el trabajo. Por supuesto, los errores que hayan quedado son de la exclusiva responsabilidad del autor. 
gramas lineales”, Representaciones. Revista de Estudios sobre Representaciones en Arte, Ciencia y Filosofía, 2 (2): 61-88.

Esquisabel, O. M. (2012), "Representing and Abstracting: An Analysis of Leibniz's Concept of Symbolic Knowledge", en A. Lassalle Casanave (comp.), Symbolic Knowledge from Leibniz to Husserl (Londres: College Publications, 1-49).

Fabbrichesi, R. (2011), "Iconic Thought and Diagrammatical Scripture: Peirce and the Leibnizian Tradition”, Semiotica, 186: 111-127. DOI: 10.1515/semi.2011.048

Fisch, M. H. (1986), “Peirce and Leibniz”, en K. L. Ketner y C. J. W. Kloesel (comps.), Peirce, Semeiotic and Pragmatism (Bloomington: Indiana University Press, 249-260).

Gregory, D. F. (1839), "On the Real Nature of Symbolical Algebra”, Transactions of the Royal Society of Edinburgh, 14 (1): 208-216.

Ketner, K. L. y Kloesel, C. J. W. (1986) (comps.), Peirce, Semeiotic and Pragmatism (Bloomington: Indiana University Press).

Lambert, J. H. (1764), Neues Organon oder Gedanken über die Erforschung und Bezeichnung des Wahren und dessen Unterscheidung vom Irrthum und Schein. (Leipzig). Reproducido en J. H. Lambert, Philosophische Schriften. 10 Bde. in 13 Bdn. Begonnen von Hans Werner Arndt, vols, I y II (Hildesheim: Georg Olms, 1965).

Lassalle Casanave, A. (2012) (comp.), Symbolic Knowledge from Leibniz to Husserl (Londres: College Publications).

Legris, J. (2012), "Between Calculus and Semantic Analysis: Symbolic Knowledge in the Origins of Mathematical Logic", en A. Lassalle Casanave (comp.), Symbolic Knowledge from Leibniz to Husserl (Londres: College Publications, 79-113).

Legris, J. (2015), "On Universality and Formality in 19th Century Symbolic Logic: the Case of Schröder's 'Absolute Algebra'” en A. Koslow y A. Buschbaum, The Road to Universal Logic. Festschrift for the 50th Birthday of Jean-Yves Béziau Volume I (Basel: Birkhäuser: 351-359). DOI: 10.1007/978-3-319-10193-4

Legris, J. (2017), "Peirce's Diagrammatic Logic and the Opposition between Logic as Calculus vs. Logic as Universal Language”, Revista Portuguesa de Filosofia, 73 (3-4): 1095-1114. DOI: 10.17990/RPF/2017_73_3_1095

Legris, J. (2018), "Existential Graphs as a Basis for Structural Reasoning”, en P. Chapman, G. Stapleton, A. Moktefi, S. Pérez-Kriz, y F. Bellucci (comps.), Diagrammatic Representation and Inference (Cham: Springer, 590-597). DOI: 10.1007/978-3-31991376-6

Leibniz, G. W. (C), Opuscules et fragments inedits de Leibniz, compilados por Louis Couturat (París: Felix Alcan, 1903).

Leibniz, G. W. (Erdmann), Opera philosophica, editada por J. E. Erdmann (Berlín: 1840, reimpresión Aalen: 1959).

Liszka, J. (1978), “Community in C. S. Peirce: Science as a Means and as an End”, Transactions of the Charles S. Peirce Society, 14 (4):305-321. URL = <http://www.jstor. org/stable/40319853>.

Peacock, G. (1834), "Report on the Recent Progress and Present State of Certain Branches of Analysis", en Report of the Third Meeting of the British Association for the

JAVIER LEGRIS - La tradición del conocimiento simbólico en el pensamiento de... I 253-271 
Advancement of Science held at Cambridge in 1833 (Londres:John Murray, 185-352).

Peckhaus, V. (1997), Logik, Mathesis universalis und allgemeine Wissenschaft (Berlín: Akademie).

Peirce, C. S. (CP), Collected Papers. 8 vols., vols. $1-6$ editados por Ch. Hartshorne y P. Weiss, vols. 7-8 editados por A.W. Burks (Cambridge: Harvard University Press, 1931-1958).

Peirce, C. S. (M), I. Manuscripts. Charles S. Peirce papers, MS Am 1632, Houghton Library, Harvard College Library. URL $=<$ https://id.lib.harvard.edu/ead/c/ hou02614c00001/catalog>.

Peirce, C. S. (NEM), The New Elements of Mathematics, 4 vols., editados por C. Eisele (La Haya: Mouton, 1976; New Jersey: Atlantic Highlands, 1976).

Peirce, C. S. (OFR), Obra Filosófica Reunida, vols. I y II, traducción de D. McNabb, revisada por S. Barrena (Madrid: Fondo de Cultura Economica, 2012).

Peirce, C. S. $(W)$, Writings of Charles Sanders Peirce: A Chronological Edition, editado por M. Fisch et al., vols.1-6 (Bloomington: Indiana University Press, 1982-2010).

Pietarinen, A.-V. (2018), “The Beauty of Graphs”, en P. Chapman, G. Stapleton, A. Moktefi, S. Pérez-Kriz, y F. Bellucci (comps.), Diagrammatic Representation and Inference (Cham: Springer, 9-12). DOI: 10.1007/978-3-319-91376-6

Raffo Quintana, F. (2019), Continuo e infinito en el pensamiento leibniziano de juventud (Granada: Comares).

Raffo Quintana, F. (2020), "Sobre compendios y ficciones en el pensamiento juvenil de Leibniz”, Revista Latinoamericana de Filosofía, 46 (1):131-150. DOI: 10.36446/ rlf2020203.

Roberts, D. (1973), The Existential Graphs of Charles. S. Peirce (La Haya: Mouton)

Schärfe, H., Hitzler, P. y Øhrstrøm, P. (2006) (comps.), Conceptual Structures: Inspiration and Application. ICCS 2006. Lecture Notes in Computer Science 4068 (Springer: Berlín - Heidelberg).

Schröder, E. (1890), Vorlesungen über die Algebra der Logik, Bd.1. (Leipzig, B.G.: Teubner. Reprints: Chelsea: 1966, Thomes Press: 2000).

Stjernfelt, F. (2006), "Two Iconicity Notions in Peirce's Diagrammatology" en H. Schärfe, P. Hitzler y P. Øhrstrøm (comps.), Conceptual Structures: Inspiration and Application (Springer: Berlin - Heidelberg, 70-86). DOI: 10.1007/11787181_6

Recibido: 16-08-2019; aceptado: 17-03-2020 